\title{
Analysis on How to Locate the Maximum Line Voltage to Hull in Steady State on the Vector Diagram Onboard Vessels
}

\author{
Soon-Man $\mathrm{Choi}^{\dagger}$ \\ (Received November 16, 2011; Revised November 22, 2011; Accepted November 28, 2011)
}

\begin{abstract}
Power distribution onboard vessel is typically configured as ungrounded system due to the ability to continuously supply electric power even when an earth fault occurs. The impedance connections between 3 phase power lines and hull cause the line-to-hull voltages to become unstable and increased in case the impedances are unbalanced, bringing the situation susceptible to electric shock and deterioration of insulation material. Also the line-to-hull voltage can reach to a certain maximum value in the steady state depending on the distributed capacitances and grounding resistances between lines and hull. This study suggests how to find and calculate the maximum line-to-hull voltage in view of magnitude and phase angle based on the vector diagram.
\end{abstract}

Key words : Ungrounded power system, Line to ground voltage, neutral point, distributed capacitance

\section{Introduction}

Onboard vessels, ungrounded power distribution systems are usually adopted to avoid the risk of service failure at the time of an earth fault. But the fault brings uprising line to hull voltages on the healthy phases and more stressful situation to their insulation materials [1-3] and various studies have been conducted for fault protection and detection [4-5].

In case of an earth fault, the deformed feature of line-to-hull voltages on the vector diagram can be reasonably described by positioning the neutral point which varies according to the insulation resistances and distributed capacitance between electric wires and hull [6-7]. One doubt about lineto-hull voltages on the real systems is that the measurement at healthy phase sometimes indicates high voltages even surpassing the line-to-line voltage under a certain condition of earth fault which is not in resonant or intermittent grounding. The detailed cause of this surpassing high voltage in the steady state has not been known so far even if it is important when considering the ungrounded power distribution systems. But it is expected that the reason of this feature could be analyzed and disclosed if the neutral point at this indication on the vector diagram is found.

This study focuses on how to get the neutral point which brings the peak line-to-hull voltage based on the loci patterns of neutral points. And its peak value and phase angle from calculation will be compared with the measured results at a real distribution system for experiment.

\section{Neutral point on vector diagram 2.1 Vector of neutral point}

Figure 1 is a schematic circuit to describe the situation of the power distribution lines onboard a

\footnotetext{
† Corresponding Author (Korea Institute of Maritime and Fisheries Technology, E-mail: mindmind@chol.com, Tel: :051-620-5850)
}

This paper is extended and updated from the short version that apeared in the Proceedings of the International symposium on Marine Engineering and Technology (ISMT 2011), held at BEXCO, Busan, Korea on October 25-28, 2011. 
ship which are linked by insulation resistances $R_{R}$, $R_{S}, \quad R_{T}$ for each phase and distributed capacitances $C$ between power lines and hull. Here, $\dot{V}_{R}, \dot{V}_{S}$ and $\dot{V}_{T}$ are phase voltages and ' $\mathrm{N}$ ' indicates the neutral point at healthy condition. If a power line is grounded, the neutral point ' $\mathrm{N}$ ' is moved to 'n' by $\dot{V}_{n N}$ as shown in Figure 2 .

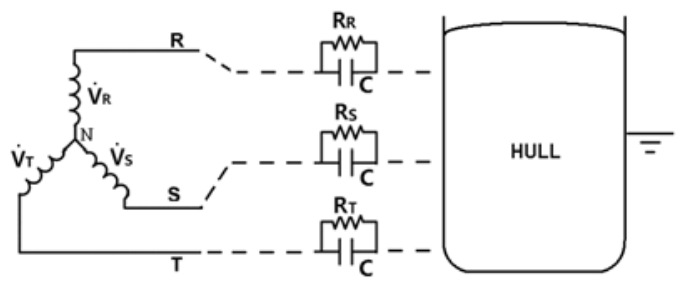

Figure 1: Schematic circuit of power lines to hull

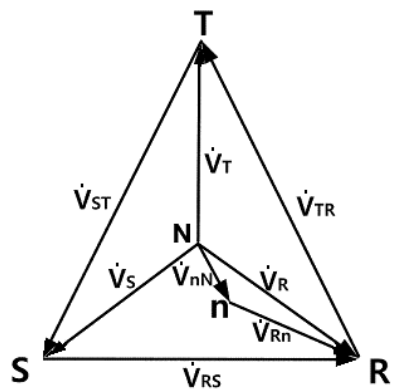

Figure 2: Neutral point ' $\mathrm{n}$ ' on the vector diagram

To know the position of neutral point ' $n$ ' on the vector diagram, we can use the equation (1), (2) and (3) based on the relation of the schematic circuit.

$$
\begin{aligned}
& \dot{V}_{R}=\dot{V}_{R n}+\dot{V}_{n N} \\
& \dot{V}_{S}=\dot{V}_{S n}+\dot{V}_{n N} \\
& \dot{V}_{T}=\dot{V}_{T n}+\dot{V}_{n N}
\end{aligned}
$$

The line-to-hull voltages $\dot{V}_{R n}, \dot{V}_{S n} \dot{V}_{T n}$ of the above corresponds to the product of the leakage current to the ground and the impedances through which they flows at each phase, and we get equation (4), (5) and (6).

$$
\dot{V}_{R n}=\dot{I}_{R} \dot{Z}_{R}
$$

$$
\dot{V}_{S n}=\dot{I}_{S} \dot{Z}_{S}
$$

$$
\dot{V}_{T n}=\dot{I}_{T} \dot{Z}_{T}
$$

Where, $\dot{I}_{R}, \dot{I}_{S}$ and $\dot{I}_{T}$ are the leakage current to hull at each phase. And $\dot{Z}_{R}, \dot{Z}_{S}, \dot{Z}_{T}$ are impedances at 3 phase lines consisting of insulation resistance and capacitance $C$ as follows.

$$
\begin{aligned}
& \dot{Z}_{R}=\frac{R_{R}\left(1-j \omega R_{R} C\right)}{1+\omega^{2} R_{R}^{2} C^{2}} \\
& \dot{Z}_{S}=\frac{R_{R}\left(1-j \omega R_{S} C\right)}{1+\omega^{2} R_{S}^{2} C^{2}} \\
& \dot{Z}_{T}=\frac{R_{T}\left(1-j \omega R_{T} C\right)}{1+\omega^{2} R_{T}^{2} C^{2}}
\end{aligned}
$$

In addition, the relation of leakage currents to hull shall be as equation (10).

$$
\dot{I}_{R}+\dot{I}_{S}+\dot{I}_{T}=0
$$

From the above equations, we get following equation as a function of impedances and $\dot{V}_{n N}$.

$$
\frac{\dot{V}_{R}-\dot{V}_{n N}}{\dot{Z}_{R}}+\frac{\dot{V}_{S}-\dot{V}_{n N}}{\dot{Z}_{S}}+\frac{\dot{V}_{T}-\dot{V}_{n N}}{\dot{Z}_{T}}=0
$$

Then, $\dot{V}_{n N}$ becomes equation (12) which allows the neutral point to be plotted on the vector diagram.

$$
\dot{V}_{n N}=\frac{\dot{Z}_{R} \dot{Z}_{S} \dot{V}_{T}+\dot{Z}_{S} \dot{Z}_{T} \dot{V}_{R}+\dot{Z}_{T} \dot{Z}_{R} \dot{V}_{S}}{\dot{Z}_{R} \dot{Z}_{S}+\dot{Z}_{S} \dot{Z}_{T}+\dot{Z}_{T} \dot{Z}_{R}}
$$

\section{2 neutral points on the complex plane}

For plotting the neutral point, the result of equation (12) needs to be described by a complex variable as shown in equation (13). 


$$
\dot{V}_{n N}=\frac{\alpha_{2}+j \beta_{2}}{\alpha_{1}+j \beta_{1}}=V_{X}+j V_{Y}
$$

Before obtaining the real part $V_{X}$ and the imaginary part $V_{Y}$ of $\dot{V}_{n N}$, it is necessary that the coefficients $\alpha_{1}, \beta_{1}$ of the denominator part of equation (12) and $\alpha_{2}, \beta_{2}$ of the numerator part are acquired from the data of impedances and the source voltages.

If these 4 coefficients are calculated from a given power system, then $V_{X}$ and $V_{Y}$ can be expressed as equation (14) and (15).

$$
\begin{gathered}
V_{X}=\frac{V_{P}}{2} \frac{\alpha_{1} \alpha_{2}+\beta_{1} \beta_{2}}{\alpha_{1}^{2}+\beta_{1}^{2}} \\
V_{Y}=\frac{V_{P}}{2} \frac{\alpha_{1} \beta_{2}-\alpha_{2} \beta_{1}}{\alpha_{1}^{2}+\beta_{1}^{2}}
\end{gathered}
$$

Where, $V_{P}=\left|\dot{V}_{R}\right|=\left|\dot{V}_{S}\right|=\left|\dot{V}_{T}\right|$, and $V_{P}$ is the phase voltage.

If $R_{R}, R_{S}, R_{T}$ and $C$ are given at a distribution system, each coefficient becomes as follows.

$$
\begin{aligned}
\alpha_{1}= & R_{R S} k_{R S} k_{T}+R_{S T} k_{S T} k_{R}+R_{T R} k_{T R} k_{S} \\
\beta_{1}= & -k_{1}\left\{R_{R S} R_{R+S} k_{T}+R_{S T} R_{S+T} k_{R}+R_{R S} R_{R+S} k_{T}\right\} \\
\alpha_{2}= & 2 R_{R S} k_{R} k_{T}-R_{T R}\left\{k_{R S}+\sqrt{3} k_{1} R_{T+R}\right\} k_{S} \\
& -R_{R S}\left\{k_{R S}-\sqrt{3} k_{1} R_{R+S}\right\} k_{T} \\
\beta_{2}= & k_{1}\left\{-2 R_{S T} R_{S+T} k_{R}+R_{T R}\left(R_{T+R}-\frac{\sqrt{3}}{k_{1}} k_{T R}\right) k_{R}\right. \\
& \left.+R_{R S}\left(R_{R+S}+\frac{\sqrt{3}}{k_{1}} k_{R S}-\sqrt{3} k_{1} R_{R+S}\right) k_{T}\right\}
\end{aligned}
$$

Where, $\omega$ is angular frequency with unit of $[\mathrm{rad} / \mathrm{s}]$,

$$
\begin{aligned}
& k_{1}=\omega C, k_{2}=\omega^{2} C^{2}, \\
& R_{R S}=R_{R} R_{S}, R_{S T}=R_{S} R_{T}, R_{T R}=R_{T} R_{R}, \\
& k_{R}=1+k_{2} R_{R}{ }^{2}, \quad k_{S}=1+k_{2} R_{S}{ }^{2}, \quad k_{T}=1+k_{2} R_{T}{ }^{2} \\
& k_{R S}=1-k_{2} R_{R S}, \quad k_{S T}=1-k_{2} R_{S T}, \\
& k_{T R}=1-k_{2} R_{T R}
\end{aligned}
$$

\section{Loci of neutral points}

Figure 3 is the loci of $\dot{V}_{n N}$ on the complex plane under the condition the insulation resistance at $\mathrm{R}$ phase only is being lowered gradually from $1.0[\mathrm{M} \Omega]$ to $0[\Omega]$ with constant $R_{S}$ and $R_{T}$ of 1.0 [M $\Omega]$. The lines of $\dot{V}_{R N}, \dot{V}_{S N}$ and $\dot{V}_{T N}$ represents the voltages to hull at healthy condition.

The loci $\mathrm{A}, \mathrm{B}, \mathrm{C}, \mathrm{D}$ and $\mathrm{E}$ are at cases where the capacitance $C$ for the distributed capacitance of power line system is given as $0[\mu F], 0.001[\mu F]$, $0.01[\mu F], \quad 0.1[\mu F]$ and $1.0[\mu F]$, respectively. When capacitance is zero, the locus shows it follows the real axis in the form of straight line.

In addition, it appears that the more the capacitance $C$ increases, the more the locus tends to have the shape of half circle. Here we know that curve D and E overlap each other showing same curves. This indicates that if the capacitance is higher than $0.1[\mu F]$ with the insulation condition given previously, the eventual curves become an apparent half circle with diameter $V_{R N}$ equal to the phase voltage of a 3 phase power system. The general results from various cases show the domain of neutral points is always limited inside the boundary of this half circle. It explains that the maximum line-to-hull voltage can be acquired only when the locus moves along the way of the half circle. and 


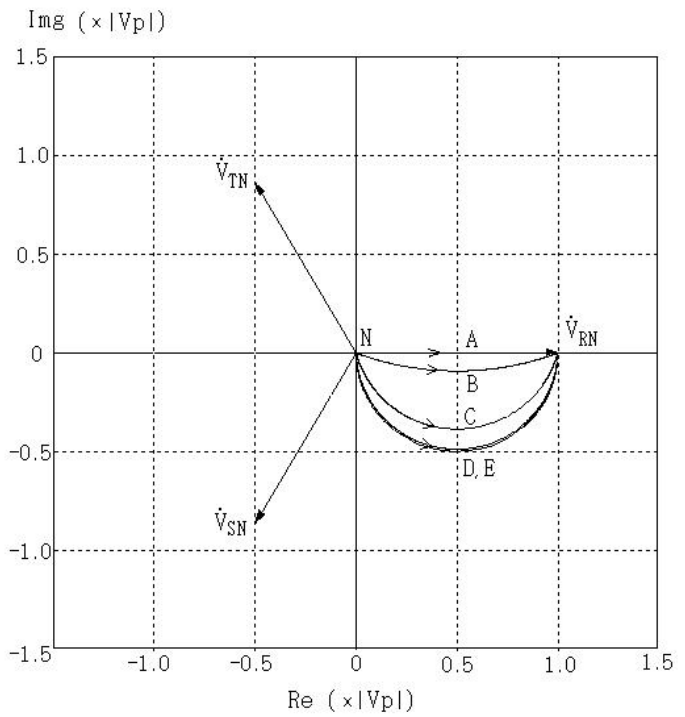

Figure 3: Loci of neutral points at 5 cases of $\mathrm{C}(0$ $[\mu F], 0.001[\mu F], 0.01[\mu F], 0.1[\mu F]$ and $1.0[\mu F])$

\section{Locating maximum line to hull voltage}

\subsection{Critical neutral point}

If the insulation resistance $R_{R}$ is lowered and the capacitance $C$ is quite big, the locus becomes the Fig. 4 where the curve of half circle shows the diameter corresponding to the phase voltage of 3 phase power lines in length. Here $\dot{V}_{T n}$, the voltage between the $T$ phase line and neutral point ' $n$ ' which is called the voltage to hull at $\mathrm{T}$ phase goes higher than the phase voltage $V_{P}$ in the whole path. In Figure 4, $\dot{V}_{T S}$ indicates $\dot{V}_{T n}$ at the time when the $\mathrm{R}$ phase line has been grounded completely with the voltage magnitude corresponding to the line to line voltage $V_{L}$ of 3 phase power system. Likewise, $\dot{V}_{T L}$ represents $\dot{V}_{T n}$ just when the magnitude of $\dot{V}_{T n}$ equals $V_{L}$ and $n_{c}$ is the neutral point of this case. On the way passing through the locus, neutral points before the critical neutral point $n_{c}$ causes $V_{T n}$ to be less than $V_{L}$. From the point $n_{c}$, there are two curves described as 1 and 2 before reaching the same point $(1, j 0)$ corresponding to completely grounded condition. Curve 2 is a drawn line for reference which makes the length $V_{T n}$ equal to $V_{L}$. From the relation of curves 1 and 2, it is obvious that the real $V_{T n}$ becomes greater than $V_{L}$ if $V_{T n}$ is between 2 lines of $\dot{V}_{T L}$ and $\dot{V}_{T S}$. The position of $n_{c}$ which makes $V_{T n}$ same as $V_{L}$ can be decided by Equation (20) and (21) based on the geometry relation.

$$
\begin{aligned}
& \left(V_{X}+\frac{V_{P}}{2}\right)^{2}+\left(V_{Y}+\frac{\sqrt{3}}{2} V_{P}\right)^{2}=\left(\sqrt{3} V_{P}\right)^{2} \\
& V_{Y}=\sqrt{V_{X}\left(V_{P}-V_{X}\right)}
\end{aligned}
$$

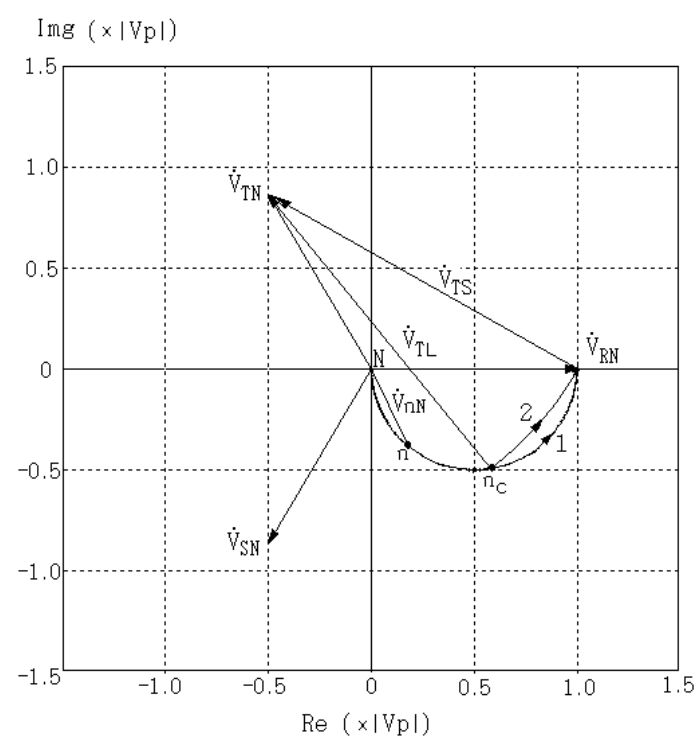

Figure 4: Critical neutral point $n_{c}$ on the plane

Then, the real part $V_{X C}$ and the imaginary part $V_{Y C}$ of $n_{c}$ are acquired as follows.

$$
\begin{aligned}
& V_{X C}=\frac{4}{7} V_{P} \\
& V_{Y C}=\frac{2 \sqrt{3}}{7} V_{P}
\end{aligned}
$$




\subsection{Maximum line to hull voltage}

The movement of neutral point ' $n$ ' from the origin to a point on the locus in Figure 4 causes $V_{T n}$ to vary in the range from phase voltage to a certain high voltage, and $V_{T n}$ experiences once becoming a peak value while $\mathrm{R}$ phase line is gradually grounded to $0[\Omega]$. If the peak $V_{T n}$ is denoted as $V_{T M}$, it shall locate between 2 lines of $\dot{V}_{T L}$ and $\dot{V}_{T S}$ as shown in Figure 5 .

The length of $V_{T n}$ in Figure 5 can be expressed by equation (24) as a function of variable $V_{X}$ and $V_{Y}$.

$$
V_{T n}=\sqrt{\left(V_{X}+\frac{V_{P}}{2}\right)^{2}+\left(V_{Y}+\frac{\sqrt{3}}{2} V_{P}\right)^{2}}
$$

And as $V_{Y}$ is a function of $V_{X}$ by equation (21), we get equation (25) for the magnitude of $\dot{V}_{T n}$ which include only one variable $V_{X}$.

$$
V_{T n}=\left[V_{P}^{2}+V_{P} \sqrt{3 V_{X}\left(V_{P}-V_{X}\right)}+2 V_{P} V_{X}\right]^{\frac{1}{2}}
$$

Then, $V_{T M}$, the peak $V_{T n}$, can be found when the derivative of equation (26) becomes zero.

$$
\frac{d\left[V_{T n}\right]}{d V_{X}}=0
$$

If $n_{m}$ is denoted as the neutral point which bring $V_{T M}$ and its real part and the imaginary part are defined as respectively $V_{X M}$ and $V_{Y M}$, the result is shown as follows.

$$
\begin{aligned}
& V_{X M}=0.75 V_{P} \\
& V_{Y M}=\frac{\sqrt{3}}{4} V_{P}
\end{aligned}
$$

Figure 5 describes how $V_{T M}$ and $n_{m \text { are located }}$ on the vector diagram in relation with $\dot{V}_{T L}$ and $\dot{V}_{T S}$.

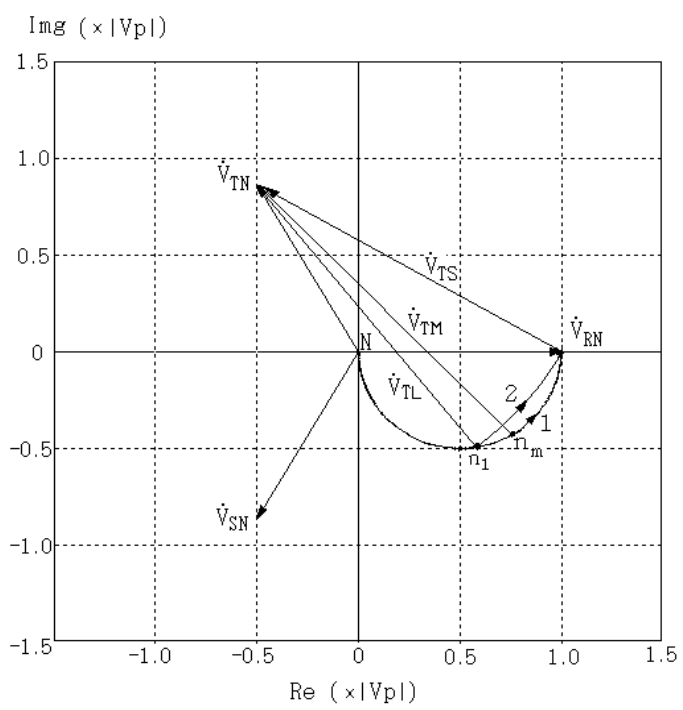

Figure 5: Maximum line to hull voltage

And if $V_{X M}$ is given to equation (29) instead of $V_{X}, V_{T M}$ is acquired as follows.

$$
V_{T M}=\frac{\sqrt{13}}{2} V_{P} \approx 1.8 V_{P}
$$

This result indicates that $V_{T M}$ becomes 1.8 times the phase voltage and even exceeds $V_{L}$ called lineto-line voltage as much as $4 \%$ higher than that in the steady state. The phase angle $\phi_{M}$ at $V_{T M}$ from the origin becomes equation (30).

$$
\phi_{M}=\tan ^{-1} \frac{j V_{Y M}}{V_{X M}}=-\frac{\pi}{6}[\mathrm{rad}]
$$

\section{Experiments and discussion}

\subsection{Experimental procedures}

Figure 6 is the configuration for experiments where 3 volt meters $V_{1}, V_{2}$ and $V_{3}$ were prepared 
for measuring $V_{R n}, V_{S n}$ and $V_{T n}$. The base metal in the schematic diagram which is a kind of power distribution console was used for the ground purpose instead of ship hull. And three miler condensers of $0.1[\mu \mathrm{F}]$ were connected for $C_{R}, C_{S}$ and $C_{T}$ between each power lines and the base metal for the effect of distributed capacitance. Here, the line to line voltage $V_{L}$ of the 3 phase power line is $220[\mathrm{~V}]$ and the phase voltage $V_{P}$ becomes 127[V]. For $R_{S}$ and $R_{T}$, two solid resistors of $1[\mathrm{M} \Omega]$ were used and one rheostat was connected for $R_{R}$ to adjust $\mathrm{R}$ phase insulation resistance from $1.0[\mathrm{M} \Omega]$ to $0[\Omega]$. To find how the line-to-hull voltage at each phase are affected according to earth condition at one phase, $R_{R}$ was decreased gradually by the rheostat and the indication of $V_{1}, V_{2}$ and $V_{3}$ were measured and recorded. Here, five measuring cases denoted as 'a', 'b', 'c', 'd', 'e', ' $f$ ' and ' $g$ ' were chosen to compare the measuring values with calculated values. The ' $a$ ' case was selected to compare both results at healthy condition where $V_{X}$ of neutral point is zero.

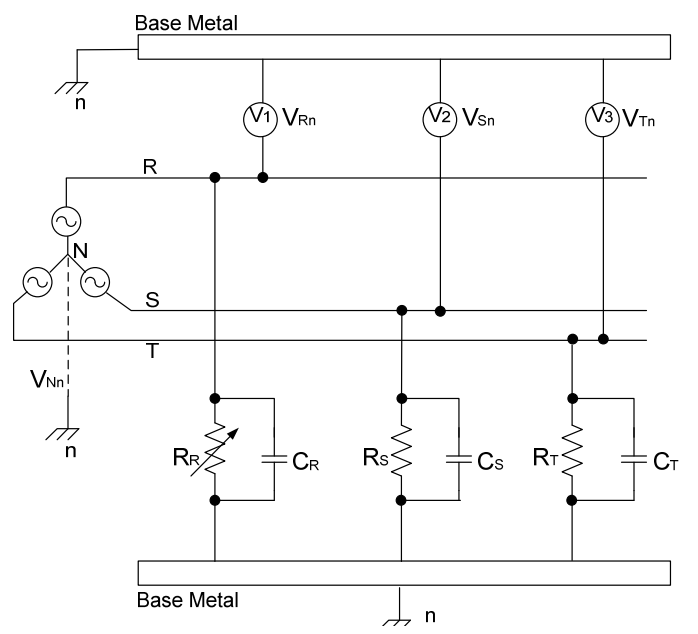

Figure 6: Schematic diagram for experiment
The value $V_{X}$ at ' $\mathrm{b}$ ' case is when it is $0.25 V_{P}$, and 'c' case is for $V_{X}=0.5 V_{P}$. The ' $\mathrm{d}$ ' case was chosen where $V_{T n}$ becomes $V_{T L}$. And at 'e' case, the values of $V_{T M}$ can be checked. The 'e' case is when $V_{X}$ is $0.75 V_{P}$ and case ' $\mathrm{g}$ ' corresponds to the completely grounded condition.

\subsection{Results of measurement}

From equation (13) and (24), the value of $V_{T n}$ is acquired by calculation if $R_{R}, R_{S}, R_{T}$ and $C$ is given. Here, it is possible to adjust the rheostat to cause the indication of volt meter $V_{3}$ to become the same value as the calculated $V_{T n}$ at each case of 'a', 'b', 'c', 'd', 'e', 'f' and 'g'. After the rheostat was adjusted to match the indication of $V_{3}$ with the calculated $V_{T n}$, then the rheostat resistances were measured as $\bar{R}_{R}$. And the indication of volt meter $V_{1}, V_{2}$ and $V_{3}$ were also recorded as $\bar{V}_{R n}, \bar{V}_{S n}$ and $\bar{V}_{T n}$ as described in Table 1.

Table 1 Values of calculation and measurement $\left(C_{R}\right.$ $\left.{ }_{=} C_{S}=C_{T}=0.1[\mu \mathrm{F}]\right)$

\begin{tabular}{|c|c|c|c|c|c|}
\hline & $\begin{array}{lll}R_{R}, & R_{S}, & R_{T} \\
\bar{R}_{R}, & \bar{R}_{S}, \bar{R}_{T}\end{array}$ & $\begin{array}{l}V_{R n} \\
\overline{V_{R n}}\end{array}$ & $\begin{array}{l}V_{S n} \\
\bar{V}_{S n}\end{array}$ & $\begin{array}{l}V_{T n} \\
\overline{V_{T n}}\end{array}$ & $V_{X}, V_{Y}$ \\
\hline $\mathrm{a}$ & $\begin{array}{l}1[\mathrm{M} \Omega], 1[\mathrm{M} \Omega], 1[\mathrm{M} \Omega] \\
1[\mathrm{M} \Omega], 1[\mathrm{M} \Omega], 1[\mathrm{M} \Omega]\end{array}$ & $\begin{array}{l}127.0 \\
127.3\end{array}$ & $\begin{array}{l}127.0 \\
127.7\end{array}$ & $\begin{array}{l}127.0 \\
127.2\end{array}$ & 0 \\
\hline b & $\begin{array}{l}15.32[\mathrm{k} \Omega], 1[\mathrm{M} \Omega], 1[\mathrm{M} \Omega] \\
14.40[\mathrm{k} \Omega], 1[\mathrm{M} \Omega], 1[\mathrm{M} \Omega]\end{array}$ & $\begin{array}{l}109.2 \\
108.7\end{array} \mid$ & $\begin{array}{l}110.8 \\
111.2\end{array}$ & & $31.7,-53.3$ \\
\hline $\mathrm{c}$ & $\begin{array}{l}8.76[\mathrm{k} \Omega], 1[\mathrm{M} \Omega], 1[\mathrm{M} \Omega] \\
8.62[\mathrm{k} \Omega], 1[\mathrm{M} \Omega], 1[\mathrm{M} \Omega]\end{array}$ & \begin{tabular}{|l|}
88.6 \\
89.1
\end{tabular} & $\begin{array}{l}135.8 \\
135.4\end{array}$ & $\begin{array}{l}213.7 \\
214.2\end{array}$ & $63.5-61.8$ \\
\hline $\mathrm{d}$ & $\begin{array}{l}7.56[\mathrm{k} \Omega], 1[\mathrm{M} \Omega], 1[\mathrm{M} \Omega] \\
7.72[\mathrm{k} \Omega], 1[\mathrm{M} \Omega], 1[\mathrm{M} \Omega]\end{array}$ & $\begin{array}{l}81.8 \\
82.3\end{array}$ & & & $72.6,-61.2$ \\
\hline e & $\begin{array}{c}4.99[\Omega], 1[\mathrm{M} \Omega], 1[\mathrm{M} \Omega] \\
5.2[\Omega], 1[\mathrm{M} \Omega], 1[\mathrm{M} \Omega]\end{array}$ & $\begin{array}{l}63.1 \\
63.8\end{array}$ & $\begin{array}{l}168.2 \\
167.1\end{array}$ & $\begin{array}{l}228.7 \\
229.8\end{array}$ & $95.3,-53.3$ \\
\hline $\mathrm{f}$ & $\begin{array}{l}3.23[\mathrm{k} \Omega], 1[\mathrm{M} \Omega], 1[\mathrm{M} \Omega] \\
3.15[\mathrm{k} \Omega], 1[\mathrm{M} \Omega], 1[\mathrm{M} \Omega]\end{array}$ & \begin{tabular}{|l|}
43.3 \\
42.5
\end{tabular} \mid & $\begin{array}{l}188.0 \\
187.6\end{array} \mid$ & $\begin{array}{l}229.4 \\
229.6\end{array}$ & $111.1,-40.3$ \\
\hline $\mathrm{g}$ & $\begin{array}{l}0[\mathrm{k} \Omega], 1[\mathrm{M} \Omega], 1[\mathrm{M} \Omega] \\
0[\mathrm{k} \Omega], 1[\mathrm{M} \Omega], 1[\mathrm{M} \Omega]\end{array}$ & $\begin{array}{l}0 \\
0\end{array}$ & $\begin{array}{l}220.0 \\
221.2\end{array}$ & $\begin{array}{l}220.0 \\
220.6\end{array}$ & $127.0,0$ \\
\hline
\end{tabular}


The result of Table 1 shows that the measuring values at experiments indicate nearly the same as the calculated values each other in all cases from ' $a$ ' to ' $g$ '. On the right column of Table 1, the calculated values of $V_{X}$ and $V_{Y}$ have been added to show the position of neutral points for each case.

The neutral points at Table 1 are also described in Figure 7 where the neutral point marked ' $c$ ' is the lowest point on the locus way. At this point, it was supposed that $V_{X}$ and $V_{Y}$ should be same if the locus is true half circle. But, it appears that $V_{Y}$ is less than $V_{X}$ indicating that the locus is slightly not a true half circle maybe because the capacitances of connected condensers are not enough to become a true half circle.

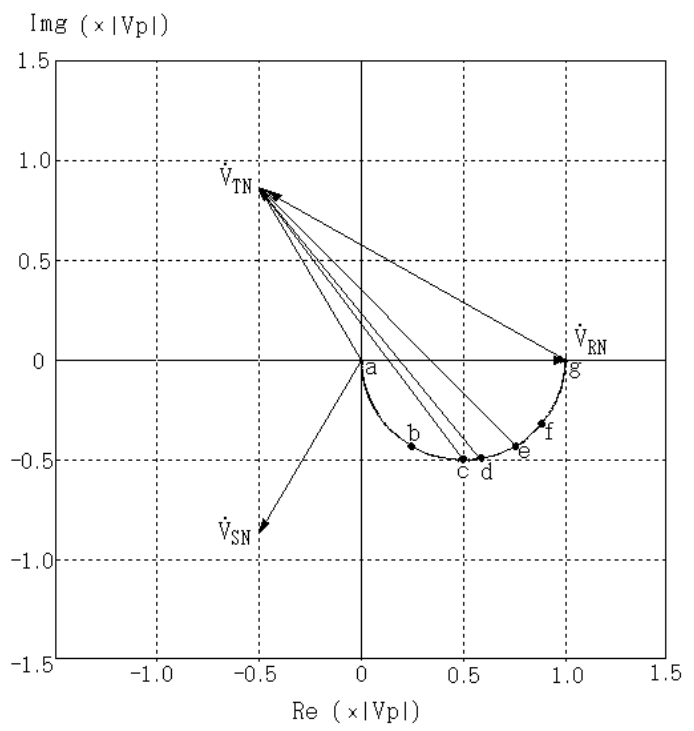

Figure 7: Neutral points corresponding to Table 1

The maximum line-to-hull voltage was expected at case ' $\mathrm{e}$ ' but in calculation values it is found at case ' $f$ '. The reason of this deviation could be explained from the fact that the locus is slight not a true half circle. But in both measuring values and calculated value, the maximum line-to-hull voltages indicates the value is $4 \%$ higher than $V_{L}$ the lineto-line voltage.

\section{Conclusions}

This paper suggested how to find the maximum line-to-hull voltage in steady state rather than at transient condition when an earth fault occurs at power distribution system. The neutral point having this max line-to-hull voltage could be found on the path which corresponds to the locus of a half circle while a power line is being grounded for analysis. The result showed that the line-to-hull voltage can increase up to 1.8 times the phase voltage which even exceeds line-to-line voltage as much as $4 \%$ higher than that in steady state based on the vector diagram.

But the position of maximum line-to-hull voltage in the calculated values was found to be slightly deviated from the expected one. The reason of this result is considered to be because the locus was not a true half circle under the chosen data.

\section{References}

[1] G.O. Watson, "Marine Electrical Practice", 5th edition, Butterworth \& Co Ltd, 1981.

[2] M.O. Durham and R. Durham, "Grounding system design for isolated locations and plant systems”, IEEE Trans. Ind. Applications., vol. 33, pp. 374-382, 1997.

[3] Kingrey, L.J., Painter, R.D., Locker, A.S., "Applying high resistance neutral grounding in medium voltage systems", IEEE transactions on industry applications, vol. 47, no. 3, pp. 12201231, 2011.

[4] S. Hanninen, M. Lehtonen, E. Antila, "A method for detection and location of high resistance earth faults", International conference on energy management and power delivery (EMPD'98), Singapore, Proceedings IEEE catalogue no. 98EX137, pp. 495-500, 1988. 
[5] D. Dalasta, B. G. Bailey, and N. Peach, "Ground fault protection", Proceedings IEEE Industry and General Applications Group, Industrial and commercial Power Systems conf., pp. 111-129, 1971.

[6] B. Bridger, "High-resistance grounding". IEEE Transactions on Industry Applications, vol. IA19, no. 1, pp. 15-21, 1983.

[7] Giuseppe Parise, "A summary of IEC protection against electric shock", IEEE Transactions on industry applications, vol. 34, no. 5, pp. 911-922, 1998.

\section{Author Profile}

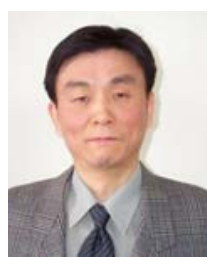

\section{Soon-Man Choi}

He received his Dr. Eng. from Korea Maritime University in 1995. He is currently a professor in the Dept. of Education and Research at Korea Institute of Maritime and Fisheries Technology in Busan, Korea. His research interests include Marine Electrical Engineering, Ship Automation Systems and Engine Room Simulators, etc. 